\title{
Harmonic detection and de-noising based on Db37 wavelet
}

\author{
WANG Qing-qing
}

\author{
Anhui University of Technology, Huainan, China
}

Keywords: Harmonic; Power quality; Wavelet transforms; Detection; Threshold de-noising

\begin{abstract}
The existence of the harmonic seriously influences the quality of the power grid electricity .In order to guarantee the safe operation of power system, it must be accurately detect harmonic and analysis harmonic components in the grid .Usually harmonic signal consists of fundamental wave, every harmonic and noise component. The noise will seriously affect the processing precision of the harmonics in power system. In this paper, the harmonic signal's detection is based on Mallat algorithm of multi-resolution analysis and the de-noising method is select wavelet threshold .Through Matlab simulation, using Db37 wavelet transform is realized on the system harmonic signal detection and separation of the signal frequency components in the system. The simulation results show that wavelet transform has good time-frequency features so it can effectively restrain and eliminate noise.
\end{abstract}

\section{Introduction}

Due to the extensive use of nonlinear load, power grid harmonic pollution becomes more and more serious [1].In order to guarantee the safe operation of power grid, it is necessary to detect harmonic in the grid. Harmonic detection is the base of deal with harmonic problems. The simplicity and effectiveness of Fourier transform (FFT) algorithm make it become the main method of years for harmonic detection. Because of FFT has the disadvantage of "stockade effect" and "spectral leakage".

Because wavelet transform's time-frequency window can adaptive change and has good time-frequency localization characteristics. It is applicable to analysis of steady state and transient time-varying signal [3]. The first task of wavelet analysis is to select wavelet function. Due to Daubechies wavelet series has the orthogonality that the power grid harmonic analysis requirements, tight branch and may be discretion features, so it has been widely used in power system harmonic analysis [4]. And due to series of Daubechies wavelet order as the time increases meanwhile, vanishing moments order number increases, the effect of frequency division is greater, so this paper uses the Db37 wavelet. Harmonic detection method based on wavelet analysis is mainly based on Mallat algorithm, wavelet packet transform and continuous wavelet transform (ntinuous wavelet transform, CWT) and so on. These methods are based on the wavelet transform which have their own characteristics and applicable situation [5].But Mallat algorithm of harmonic extraction is one of the most widely used at present.It has a good real-time dynamic and its fundamental component and harmonic component after isolated has good measuring precision.

This paper summarizes the basic principle of wavelet transform and the advantage of harmonic detection. From this obtain harmonic information and use wavelet threshold de-noising method to filter the white noise in the signal processing. In the Matlab simulation verified the effectiveness of the wavelet transform used to harmonic detection and de-noising.

\section{The Basic Principle of Wavelet Transform}

For arbitrary function $f(\mathrm{t}) \in \mathrm{L}^{2}(\mathrm{R})$ and a base wavelet $\psi(\mathrm{t}), f(\mathrm{t})$ about the function $\psi_{a, b}(\mathrm{t})$ of integral nuclear continuous wavelet transform (CWT) is defined as:

$W_{t}(\mathrm{a}, \mathrm{b})=\left\langle f, \psi_{a, b}\right\rangle=|a|^{\frac{1}{2}} \int_{-\infty}^{+\infty} f(\mathrm{t}) \psi\left(\frac{t-b}{a}\right) \mathrm{dt}$

Type: $a$ is scale factor; $b$ is translation factor. 
On the basis of CWT, a and b will be scatter to get discrete wavelet transform (DWT).Take ${ }^{a=2^{-j}, b=2^{-j} k,(j, k \in Z)}$, discrete wavelet transform is expressed as:

$$
W_{t}(\mathrm{j}, \mathrm{k})=\left\langle f, \psi_{\mathrm{j}, \mathrm{k}}\right\rangle=2^{-\frac{j}{2}} \int_{-\infty}^{+\infty} f(\mathrm{t}) \psi\left(2^{-j} \mathrm{t}-\mathrm{k}\right) \mathrm{dt}
$$

When high frequency components are decreased, the time window will narrowed automatically, the frequency window height will increased; On the contrary, the low frequency component are increased, time window will grow wider automatically, frequency window height will decreased.

\section{Based on Mallat Algorithm of Multi-resolution Analysis}

$s(t)$ set for the signal to be analysed. $s(n)$ is the discrete signal by sampling from $s(t)$.The $s(n)$ points rate analysis,

$$
a_{i+1}(n)=\sum_{k \in Z} a_{i}(k) h(k-2 n) d_{i+1}(n)=\sum_{k \in Z} a_{i}(k) g(k-2 n)
$$

$\mathrm{h}$ and g respectively as wavelet function and scaling function generated by the low-pass and high-pass filter. $a(n)$ describing the approximation part of signal after decomposition, namely the low frequency component. $d(n)$ describing the details part of signal after decomposition.

\section{The Establishment of The Harmonic Model}

In the actual power grid harmonic contains both stable every harmonic component also includes the transient change of unsteady harmonic and various noise interference. Modeling harmonic situation here is as follows:

Model 1: only contains the fundamental wave and integer harmonics

Set the model contains the corresponding 3, 5, 7 harmonic and its amplitude respectively of $1 / 3$, $1 / 5,1 / 7$ and ignore more than 7 times of high frequency harmonics. The fundamental wave amplitude is set to 1 . Signal expression is as follows:

$$
F_{1}(t)=\sin (2 \pi \cdot 50 t)+\frac{1}{3} \sin (3 \cdot 2 \pi \cdot 50 t)+\frac{1}{5} \sin (5 \cdot 2 \pi \cdot 50 t)+\frac{1}{7} \sin (7 \cdot 2 \pi \cdot 50 t)
$$

Model 2: with the noise of fundamental wave and integer harmonics

The fundamental wave contains the condition of the normal distribution of white noise and set its amplitude is 0.2, the signal expression is:

$$
F_{2}(t)=0.2 \delta(t)+\sin (2 \pi \cdot 50 t)+\frac{1}{3} \sin (3 \cdot 2 \pi \cdot 50 t)+\frac{1}{5} \sin (5 \cdot 2 \pi \cdot 50 t)+\frac{1}{7} \sin (7 \cdot 2 \pi \cdot 50 t)
$$

\section{The Results of Simulation and Analysis}

The frequency of the power grid for power frequency is 50HZ. The sampling frequency is 6.4 $\mathrm{kHz}$. Each cycle of sampling points is 128 points and continuous acquisition of five cycles, so sampling 640 points. Multi-resolution analysis using wavelet decomposition for five layers. With Db37 wavelet analysis model 1:

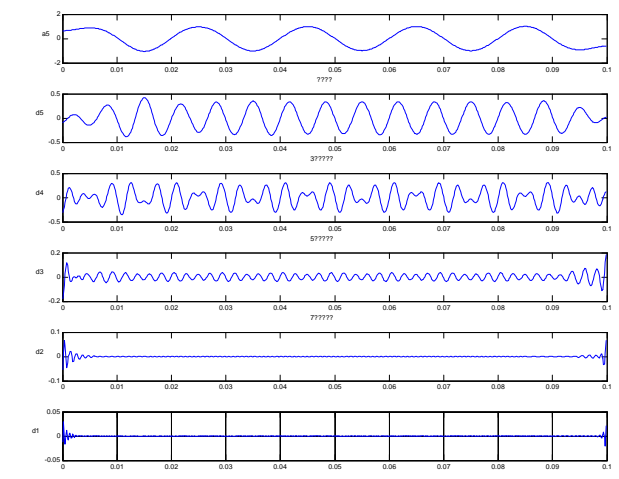

Fig.1 Contains no noise wavelet decomposition of the original signal waveform Wavelet analysis model by Db37 get fundamental wave and 3, 5, 7 harmonic. Result is shown in 
figure 1.Harmonic component by reconstructing a5, d5, d3 and d4 coefficient after Db37 wavelet transform. It can be seen from the figure the error of fundamental after wave reconstruction and the fundamental of original signal is very small. It also concludes that the reconstruction of 3, 5, 7 times harmonic component can accurately reflect the original signal harmonic frequency.

With Db37 wavelet analysis model 2:

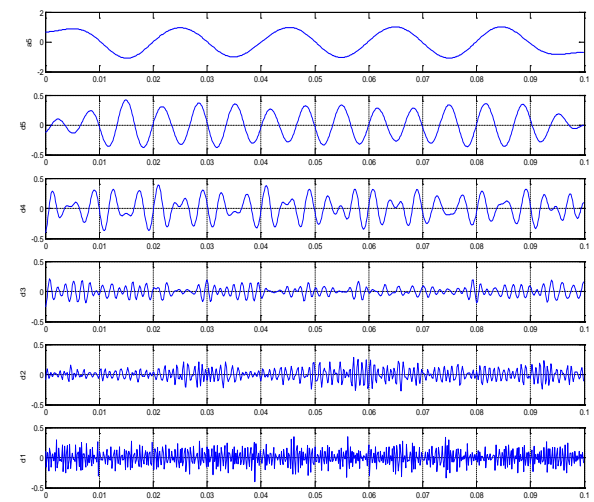

Fig.2 The original signal containing noise wavelet decomposition waveform

Figure 2 using DWT transform for the harmonic signal containing white noise F2. The figure shows that using wavelet transform method to detect the harmonic signal that containing noise. That cannot achieve the effect as shown in figure 1, then cannot effectively detect every harmonic component. This is due to the noise interference in signal detection, so it must to signal preprocessing, filter the signal of noise component, therefore it can realize accurate detection of harmonic.
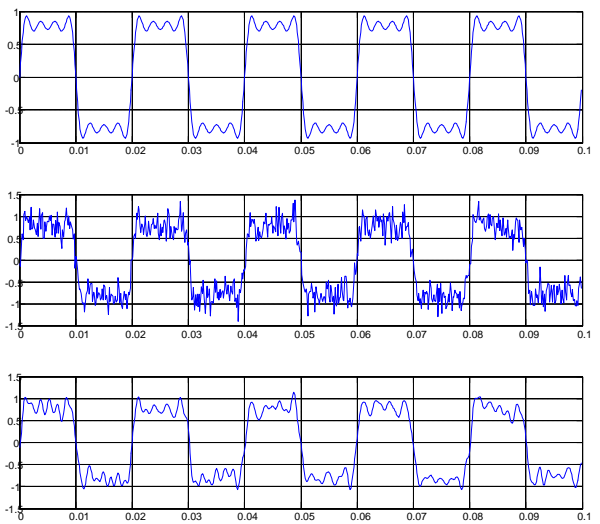

Fig.3 The default threshold denoising signal is compared with the original signal

The figure 3 Shown that the results of the default threshold de-noising signals before and after comparing. It shows that after filtered noise signal is the same as the original signal. It concluded that this method can effectively filter out noise. First the harmonic signal is decomposed discrete wavelet, moreover the noise will be first to quantify the wavelet coefficients of processing, removing noise, finally through refactoring get waveform after the noise reduction.

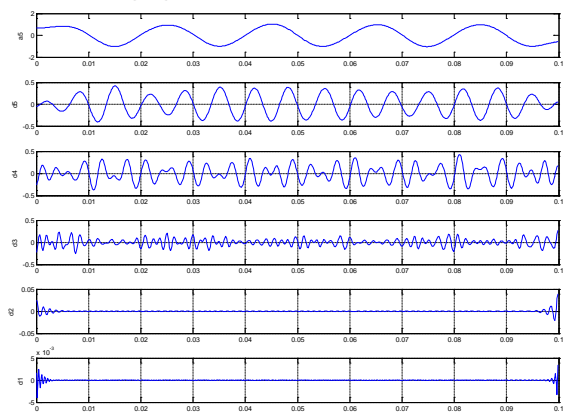

Fig.4 The waveform of wavelet decomposition after denoising signal

As shown in figure 4 is the cancellation after the noise of signal and then to the wavelet analysis, then it obtained the approximate figure 1 analysis results. Contrast figure 4 and figure 1 show that 
through the wavelet transform is effective to filter noise and basic every harmonic component was isolated.

This paper analyses the basic theory of wavelet transform and applied to harmonic detection and de-noising. The simulation shows that using wavelet transform for detecting harmonic is feasible and effective. The noise signal with wavelet transform also achieved a certain degree of noise reduction. Compared with the Fourier transform, wavelet transform is using its unique advantage_— not limited by the integral period sampling and synchronization. So it can catch to all details of the signal and more suitable for non-stationary harmonic detection and analysis. With the continuous development of wavelet theory and improvement of the wavelet transform in power system harmonic signal processing plays a more important role.

\section{Reference}

[1] Sun Cheng-xiang. The application of wavelet transform in wind power harmonic detection[D]. Xinjiang university,2008.

[2] Huang Wen-qing,Dai Yu-xing,Quan Hui-min. The harmonic analysis algorithm based on Daubechies wavelet[J]. Journal of electrotechnics, 2006,06:45-48+53.

[3] Zhou Long-hua,Fu Qing,Yu Shi-jie,Li Xiang-feng. Harmonic detection technology based on wavelet transform[J]. Electric power system and its automation, 2010,01:80-85

[4] Zhu Xiang,Jie Da,Gao Qiang,Zhang Yan-chi. Db8 wavelet application in power system harmonic analysis[J]. Journal of electric power science and technology, 2011,02:67-71.

[5] Zhou Lin,Xia Xue,Wan Yun-jie,Zhang Hai,Lei Peng. Harmonic analysis method based on wavelet transform is reviewed in this paper[J]. Journal of electrotechnics， 2006,09:67-74 